\title{
DE-EMBEDDED MEASUREMENTS USING THE HP 8510 MICROWAVE NETWORK ANALYZER
}

Glenn Elmore

Network Measurements Division

1400 Fountaingrove Parkway

Santa Rosa, CA 95401

RF \& Microwave Measurement Symposium and Exhibition

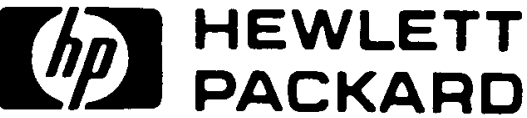

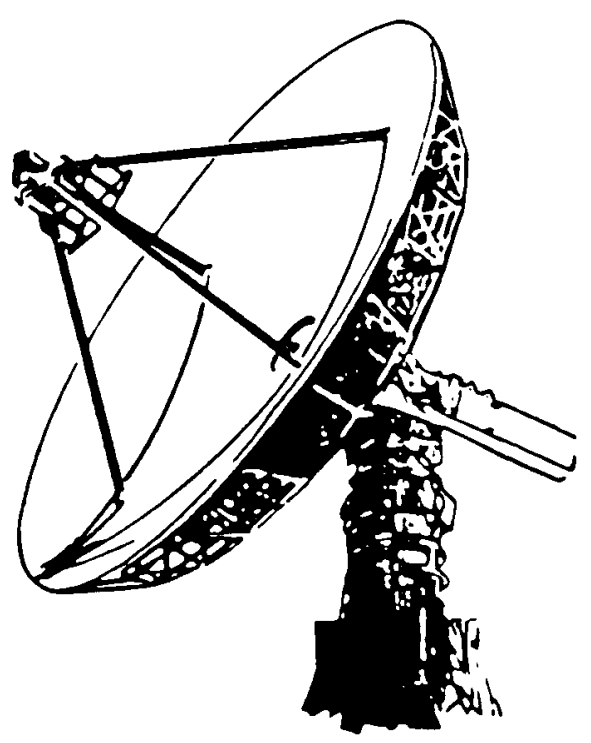




\section{DE-EMBEDDED MEASUREMENTS USING THE HP8510 MICROWAVE NETWORK ANALYZER}

This paper describes a. technique for modifying the error coefficients used inside the HP 8510A to provide a measurement vantage point different from that which normal calibration and measurement techniques will allow. Such modification enables the HP 8510A to display data as though it had been calibrated at a measurement plane separated from the actual calibration plane by an embedding network. This technique, called de-embedding, enables direct device measurement at measurement planes for which suitable calibration standards are unavailable or inconvenient to use.

While not totally general, the technique can accommodate many fixturing applications (the same ones to which conventional calibration techniques apply), particularly if certain attributes are included in the design and fabrication of the fixture.

The technique may be easily extended to allow embedding the device under test in a hypothetical network to allow viewing the device as though it were actually in a circuit with such a. network.

Some examples are given which demonstrate the measurement of a packaged transistor in a. fixture with various amounts of de-embedding. Finally, tile same transistor is shown de-embedded from the entire fixture and with a matching/filter network embedded allowing real time observation of the "finished amplifier" performance as a function of bias conditions.

Author: Glenn Elmore, R\&D Engineer, HP Network Measurements Division, Santa Rosa, CA. The author has been with HP since 1972. The majority of that time was spent as a member of a lab team developing swept microwave sources, including the HP 8350 family of broadband plug-ins. Since 1981, he has been involved with the development of the HP 8511-8515 test sets for the HP 8510 and most recently was responsible for the development of the hardware and algorithms used as part of tile HP 85014A Active Device Measurements Pac. 


\section{INTRODUCTION}

At microwave frequencies it becomes difficult to directly measure components and devices. Although automatic network analyzers such as the HP 8510 can make direct measurements when calibration standards with the same connector type as the device under test are available, many times the device cannot be connected directly to the calibration plane.

This paper describes a way to use the HP 8510 to make measurements exactly as though it had been calibrated at the plane of the device or component which is to be measured. This allows the HP 8510 to directly display the device characteristics by using its built-in error correction ability to remove known fixture influences from the measured data.

In addition, an extension of this technique is presented which allows the measurement of a real device as though it were part of a hypothetical network. This new technique, called embedding, offers a powerful blend of circuit analysis and realtime measurement.

\section{WHAT IS DE-EMBEDDING?}

Errors are a fact of life in virtually every measurement system. In the real world, any information obtained about the characteristics of a subject or device relative to some measurement standard is likely to be in error. Generally, the information available at the "output" of a measuring instrument or organ deviates from, or is a corrupted version of, tile desired information.

In general, a measurement process consists of some kind Of Stimulus or perturbation of the object of interest (located at the Device or Measurement Plane) followed by an examination of the results (obtained at the Data Collection Plane).

Tile data which is collected may be in error due to many causes; the stimulus may not be the same as that which is desired or expected, tile object (hereafter called the Device Under Test or DUT) may not be

Situated in the desired environment, other characteristics of the measuring environment may "corrupt" the collected data, and the measuring instrument at the Data Collection Plane may itself be in error or affect the measurement. 
Fortunately, it is often possible to achieve results nearer to those which are desired through analysis and correction of the "raw" data taken at the Data Collection Plane. To the degree to which the causes of error can be understood and their affects on the "actual" or desired data taken into account and corrected, the desired data may be obtainable. As the cartoon illustrates, error correction of this sort is a common Occurrence.

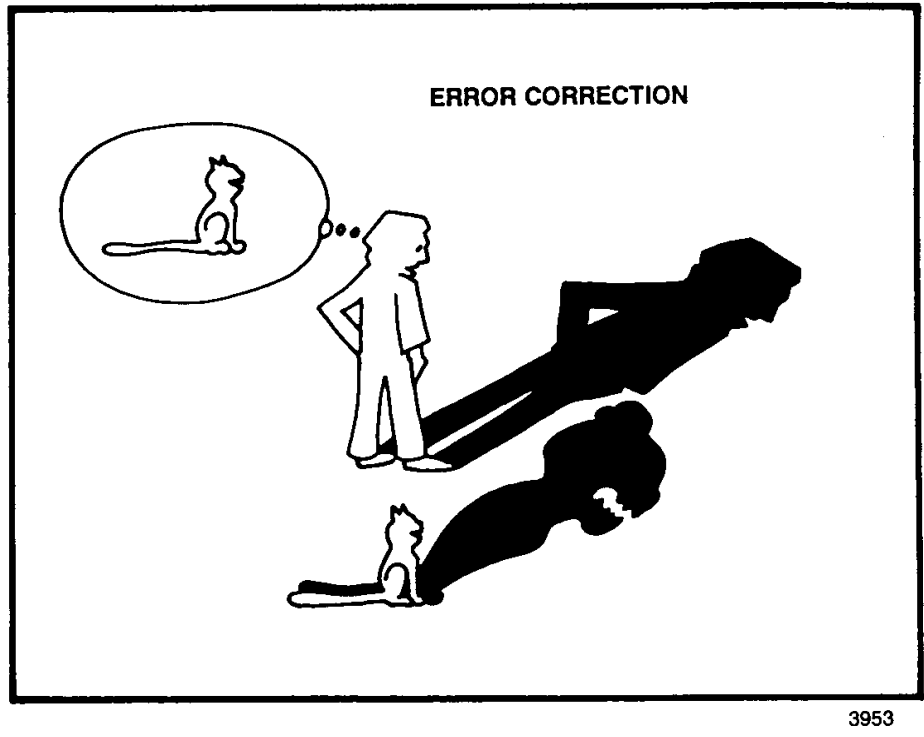

Automatic network analyzers (ANAs) perform this measurement and error correction process in the domain of network measurements. When measuring a one-port DUT, the ANA acquires data at a plane which is separated from the DUT by a corrupting network. The model of this network is given the name Error Adapter.
In order for the ANA to correct the raw data and provide the desired data, it is necessary to have a description of the Error Adapter. This must be a model of the manner in which it affects or corrupts the measurement which is valid at the time of DUT measurement. The description is often provided by a process called Calibration. Calibration consists of measuring a Sufficient number of known devices, called calibration standards, and calculating the parameters of the Error Adapter based on that raw data. Calibration is therefore the process of characterizing the Error Adapter.

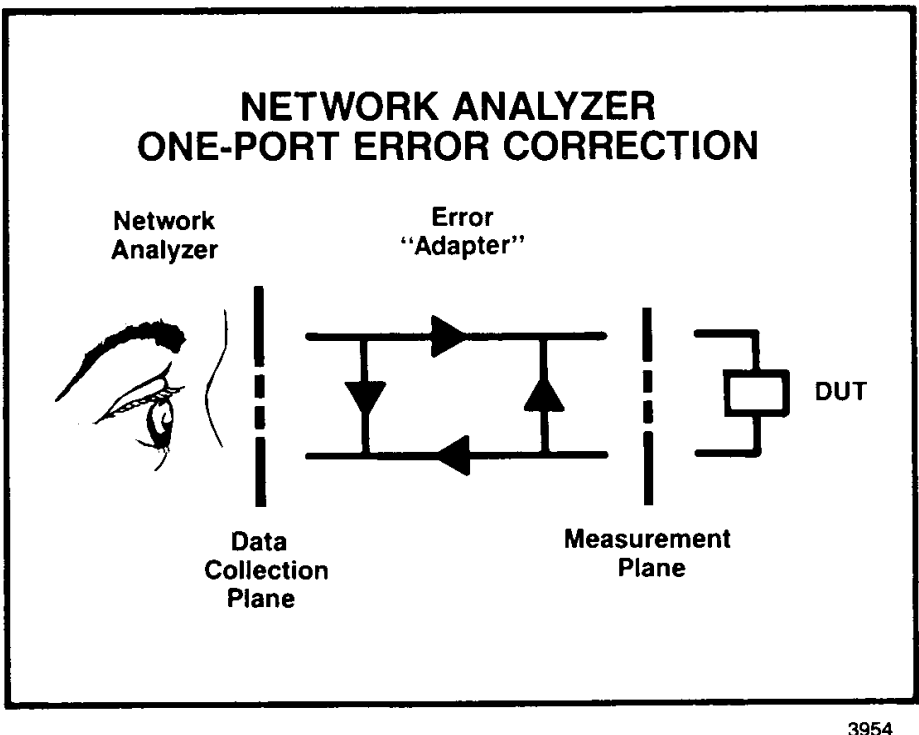

3954

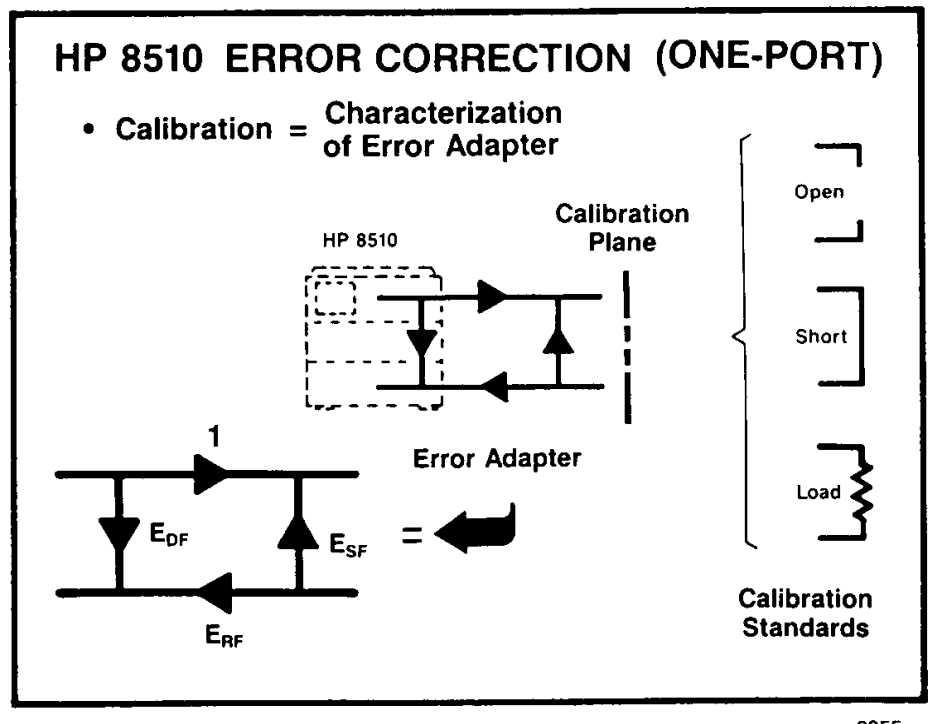

3955 


\section{HP 8510 ERROR CORRECTION (ONE-PORT)}

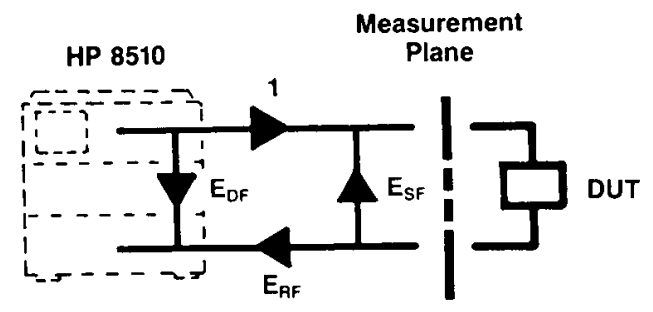

- Measurement = De-Embedding

- Measurement Plane = Calibration Plane

- HP 8510 Error Correction Process Performs De-Embedding in Real Time

\section{FIXTURED MEASUREMENTS PROBLEM}

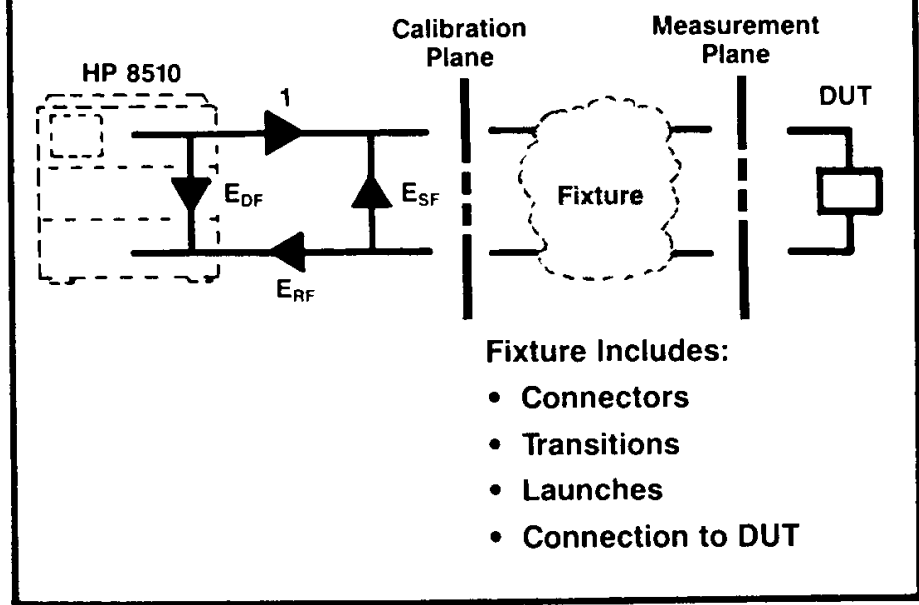

The operation of relating the characteristics of a DUT at one end of a characterized error adapter to the data taken at the other end is a bilinear transformation and has been previously described ${ }^{1}$. Earlier descriptions ${ }^{2}$ of these related processes of error adapter and DUT measurement gave names to them which were different from those currently in use. The process of error adapter characterization was called Untermination while the DUT measurement was termed de-embedding. With the HP 8510 microwave network analyzer, automatic, real-time error correction is performed by the instrument and the displayed data reflects the results Of the process. Effectively such a measurement is de-embedding according to the original definition. Similarly, the calibration process corresponds to the original definition of untermination.

It should be noted that data which results from this process is in effect data taken as though the measurement were being made at the plane established or defined by the calibration standards. Thus, for such a measurement, the measurement plane and the calibration plane are the same. The HP 8510 performs de-embedding, as previously defined, in real time.

A large class of network measurements have the attribute that the actual device which is to be measured cannot be connected directly to the connection available at the measurement, plane of a calibrated ANA. This class of DUT measurements may be termed Fixtured

Measurements, since the DUT is separated from the calibrated ANA by some kind of transitional network or fixture. An example of this is the measurement of packaged transistors. Although a structure may be devised which can serve as a transition between a coaxial connection of an ANA test set and a transistor package, Suitable calibration standards may not be available to allow a conventional calibration of the system at the plane of the transistor package. As a consequence, the measurement plane which can be obtained through calibration at the coaxial connection is separated from the DUT plane of interest by a network, the fixture. In general this fixture is not lossless and reflectionless transmission line. Rather it may be comprised of connectors. transitions between different types of transmission lines, and the connections to the DUT. Because it may have nonideal characteristics, simple techniques like reference plane extension which are available to account for the phase of an intervening ideal transmission line are not applicable. 
Consequently it may be desirable to have a way to include a description of the characteristics of the fixture along with the original error adapter characterization obtained from calibration into a description of a total or cascaded error adapter for the ANA to use. Such a combining or cascading process would provide the ability for an ANA to display corrected data of the DUT at the DUT plane, having corrected for the influences of the fixture.
INCLUDING FIXTURE ERRORS IN ANA ERROR ADAPTER

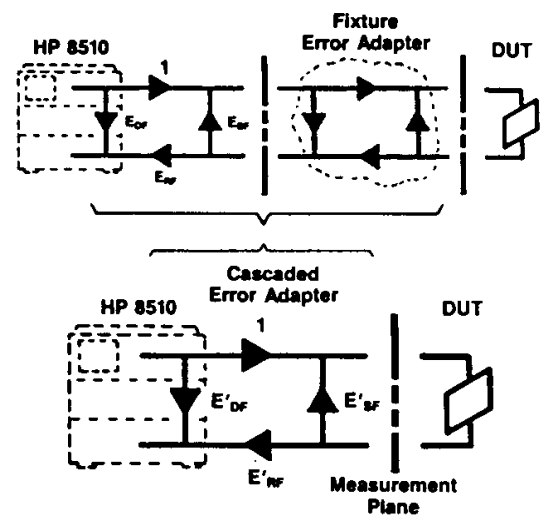

Although in one way more restrictive than the original definition ${ }^{2}$, this paper will use the term de-embedding to describe error correction by using a process to establish measurement planes different from those provided by calibration. As used here the term is applied to linear one-port or two-port measurement systems. Since it relates data at the two ends of a characterized linear two-port error adapter it may also be viewed as a bilinear transformation. It should be noted that the term de-embedding does not refer to the method used for calibrating or characterizing any of the networks between the DUT and data collection planes but only to the process of providing measurement planes different from those obtained through conventional ANA calibration with standards.

\section{DE-EMBEDDING MAY BE DESCRIBED AS:}

- Establishing New Measurement Plane Different from Calibration Plane

- Bilinear Transformation Through Error Adaptors

Note: De-Embedding Does NOT Refer to the Technique Used to Characterize the Fixture Error Adaptor 


\section{BENEFITS OF DE-EMBEDDING}

De-embedding can allow the measurement of devices which otherwise could not be directly measured. It may be useful when suitable calibration standards at the DUT plane are nonexistent as well as when such standards exist but are inconvenient to use.

In some instances conventional standards may be available but very inconvenient to use, requiring excessive disassembly of a fixture or an elaborate calibration process. If these standards can be measured only once tile data may be used to enable de-embedded measurements.

Another use for de-embedding might be the measurement of noninsertable devices ${ }^{3}$. If an appropriate adapter can be characterized, its parameters may be used in a de-embedded measurement of the DUT.

Sometimes calibration devices exist but are not of the same types required for normal ANA calibration. Measurements of these devices along with data analysis is required to extract fixture characteristics. De-embedding can be used to effectively extend the types of usable calibration techniques with the HP $8510^{7}$.

BENEFITS OF DE-EMBEDDING

De-Embedding May Be Desirable When Suitable Measurement Plane Calibration Standards:

- Aren't Convenient

- Don't Exist

3960

\section{BENEFITS OF DE-EMBEDDING WITH THE HP 8510}

- Real Time Display of DUT Measurements Human Interaction and Synthesis Yields New Insight

- Time Domain Analysis Additional DUT Information Useful to Refine Measurement
With the HP 8510, direct de-embedded measurement can provide additional information, Real time DUT data is fundamentally new information when combined with human interaction. By varying electrical/mechanical parameters such as bias, temperature, input power to DUT, or pressure and using the human ability to synthesize information, new insight about a DUT (or fixture) may be obtainable. Furthermore, time domain analysis with the HP 8510 can give additional useful DUT information. Time domain analysis is also helpful to give an indication of the degree and nature of residual fixture errors. Errors which result from fixture variation or nonrepeatability as well as errors due to imperfect characterization or modeling may be discovered. Time domain can aid in "bootstrapping" to improve the measurement of the DUT by improvements of the fixture design or characterization. 


\section{THE DE-EMBEDDING PROCESS}

In order to understand the de-embedding process, let's first look at the HP 8510A two-port error model. This model has six error terms for each direction of Stimulus. These twelve terms have been previously described in detail ${ }^{4}$. The goal of the de-embedding process is to provide error terms for an error adapter which include the fixture errors along with the error terms obtained from the calibration process. These terms must be in the same form as the calibration error terms so that the HP 8510 can properly correct the raw data.

This figure shows the original calibration terms $E_{1}[]$ and $E_{2}[$ ] being cascaded with the fixture error model terms, $\mathrm{F}_{1}[]$ and $\mathrm{F}_{2}[\mathrm{]}$ to provide new error terms, E' [ ], for the HP 8510A. The equations for the cascading operation can be written using signal flow analysis techniques ${ }^{6}$.
It should be noted that the result of the cascade of $\mathrm{E}_{\mathrm{l}}[\mathrm{]}$ (which has a unity forward transmission term) with the fixture Port 1 error model $F_{1}[$ ] produces a nonunity forward transmission term. This term must be normalized in order to satisfy the internal HP 8510A error model. This requires that the product of the cascaded transmission terms be put into E' ${ }_{\mathrm{rf}}$. Similarly, but possibly not so obviously, the forward transmission tracking term which resulted from the cascade of $F_{2}[]$ and $\mathrm{E}_{2}[]$ must be multiplied by the forward transmission term which resulted from the cascading of $\mathrm{E}_{1}\left[\mathrm{]}\right.$ and $\mathrm{F}_{1}\left[\mathrm{]}\right.$ to normalize $\mathrm{E}_{\mathrm{tf}}^{\prime}$. See Appendix A for equations describing error term modification.

\section{HP 8510A TWO-PORT ERROR MODEL}

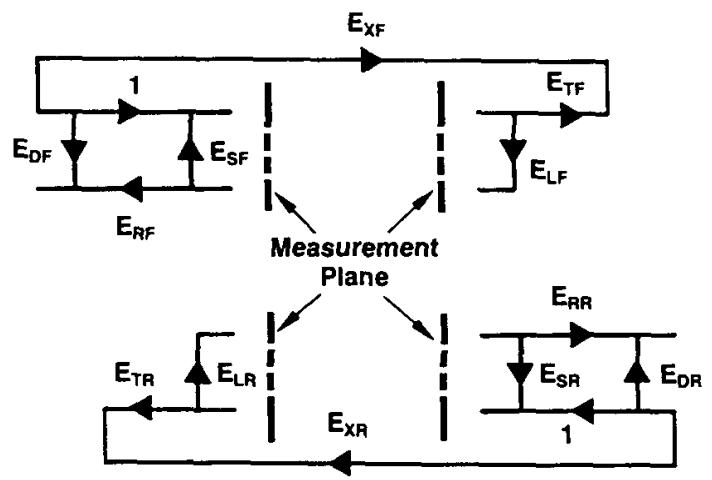

Forward

- 12 Error Terms

\section{HP 8510A TWO-PORT DE-EMBEDDING (FORWARD DIRECTION)}

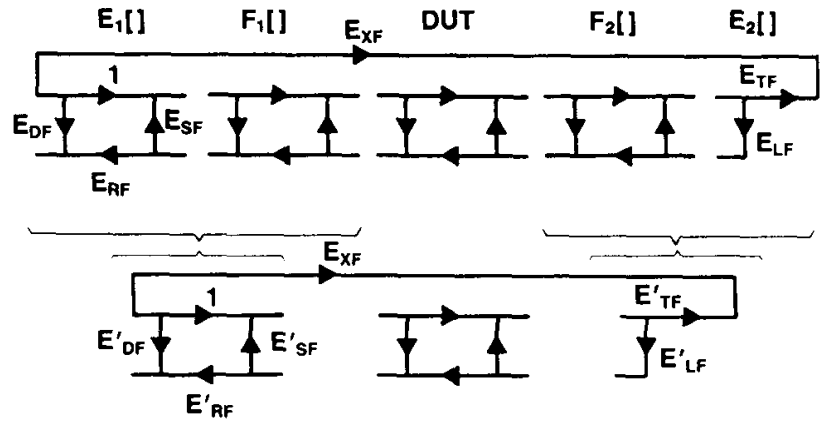

$E_{1}[], E_{2}[] \quad$ Error Terms from Calibration

$F_{1}[], F_{2}[] \quad$ Error Terms of Fixture Error Adaptor
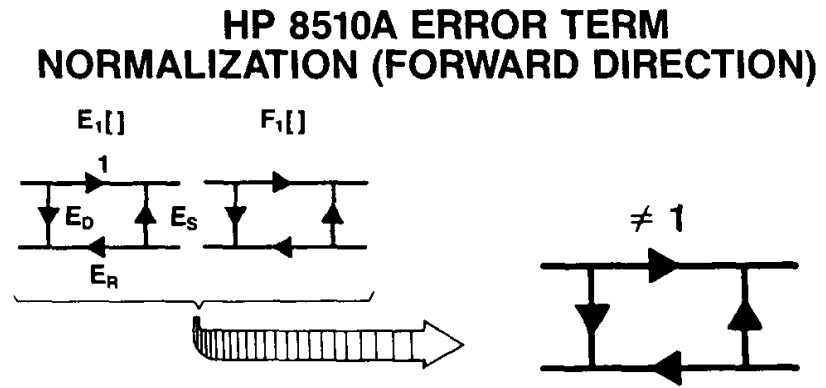

NOTICE: Cascading $E_{1}[]$ and $F_{1}[]$ Produces Non-Unity Forward Transmission Term. Thus, BOTH $E_{\mathrm{PF}}^{\prime}$ and $\mathrm{E}_{\mathrm{TF}}$ Must Be Normalized to Account for This."

*Appendix A 


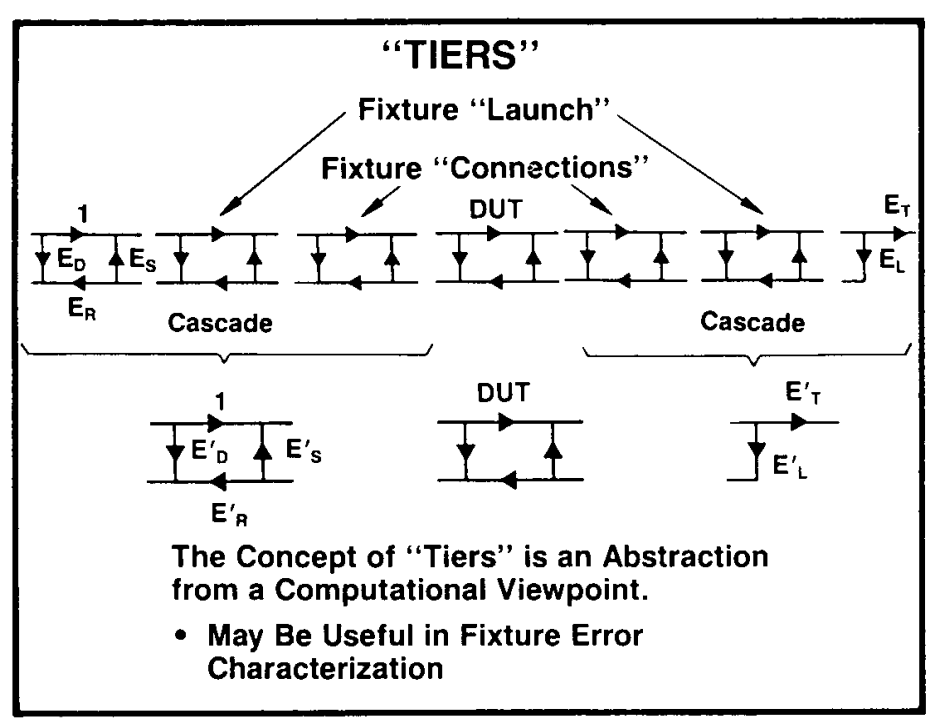

3965
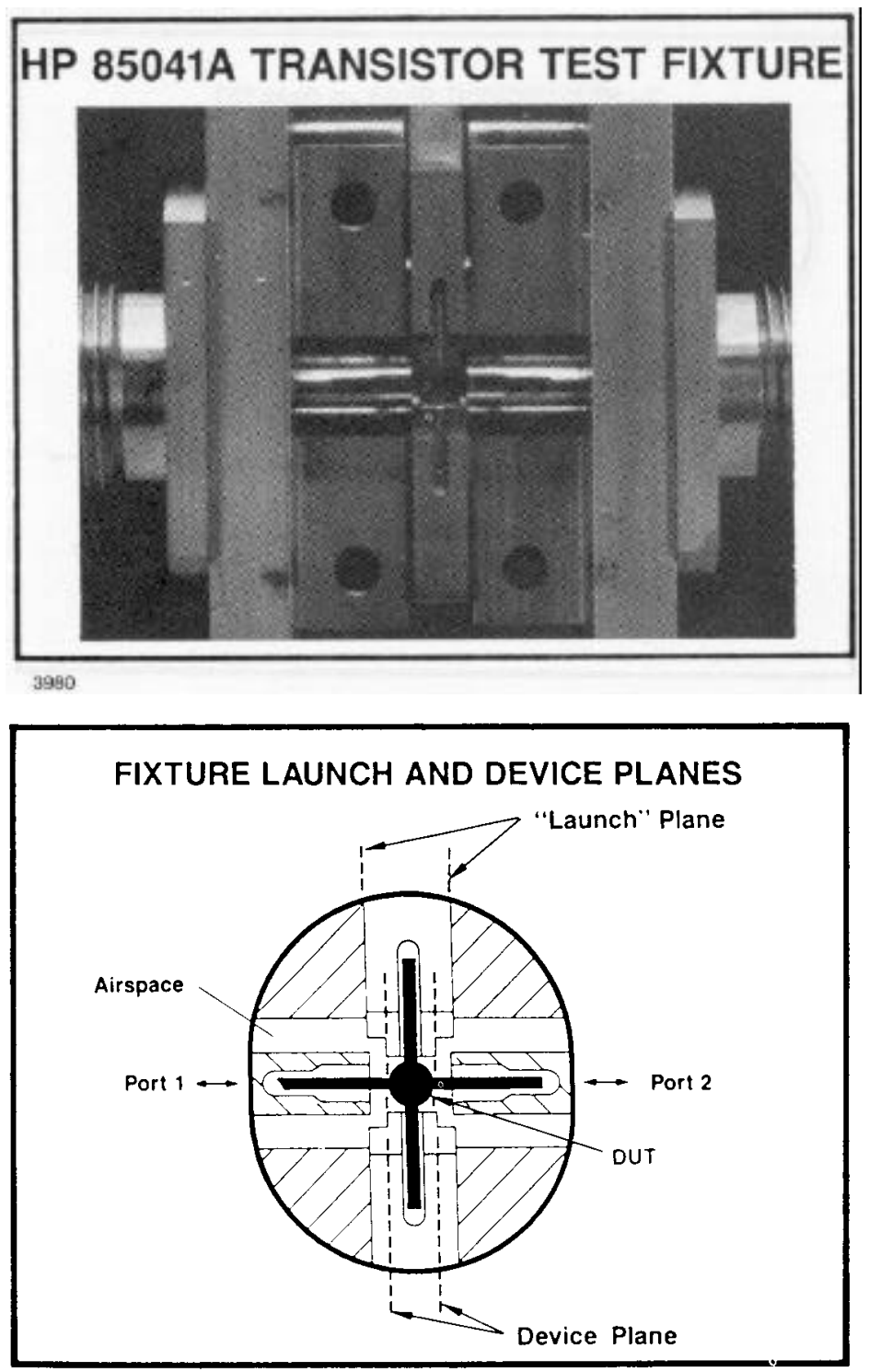

3982
A previous treatment ${ }^{5}$ of the Subject of de-embedding has presented the concept of "tiers". That is, the overall network between the collection plane and the DUT can be described as the cascade of smaller networks. This is particularly of interest when there are interconnections or physical separations within the fixture. An example is the case when a fixture has coaxial connectors for connection to the ANA at the calibration plane, and a "launch" to internal connections to the DUT, which rests in an insert. The launch and the insert may be de-embedded one at a time in order to obtain data from the DUT plane. From a computational viewpoint the result is the same whether these smaller networks are de-embedded separately or first cascaded and then de-embedded all at once. However, characterizing the smaller networks individually often gives a more physically accurate picture than might be obtained if an attempt were made to model the entire fixture at one time. This more physically accurate model sometimes can provide insight into problem areas which may be corrected to improve the overall DUT measurement.

This Picture shows a packaged transistor test fixture. $7 \mathrm{~mm}$ connectors are visible at each end and the insert in which the packaged device rests is visible in the center.

This figure illustrates the launch and device measurement planes of the previous packaged transistor test fixture. 
This figure shows the measurement of a 0.5 micron gate length gallium arsenide field effect transistor installed in an HP 85041A transistor test fixture with a $.070 \mathrm{inch}$ insert and leaded transistor package. Calibration was performed at the $7 \mathrm{~mm}$ connectors and the measurement shows the effects of the fixture and insert on the input reflection coefficient of the transistor itself.

This figure shows the same device as in the previous figure. However this is a de-embedded measurement, the error terms which were used previously have been modified to include the effects of the fixture launch. The parasitics associated with the transistor lead and insert which the transistor is mounted in, as well as the effect of the discontinuity at the fixture launch/transistor lead interface are included in the plotted data. The removal of the phase shift due to fixture length is obvious. Losses and reflections due to the fixture launch have also been removed.

In this figure, the effects of the entire fixture and insert have been included in the HP 8510 error terms. The result is a completely de-embedded measurement of the transistor at the device (DUT) plane. This data is what would be observed if a calibrated ANA could be placed right at the DUT measurement plane. The differences among the data for the three plots correspond to the measurement error contribution of the intervening networks.
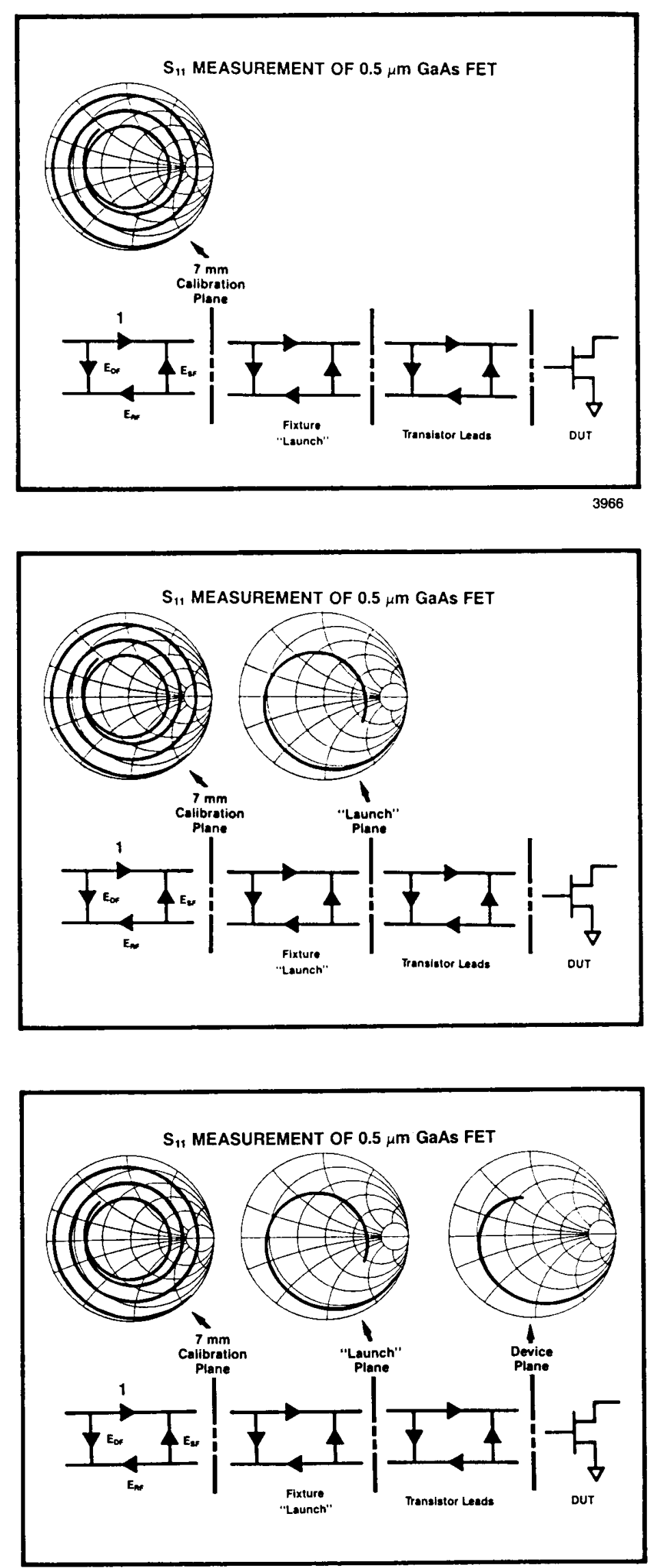


\section{FIXTURE CONSIDERATIONS}

In designing and using a fixture for the measurement of a DUT with an HP 8510 there are a number of issues to be considered to obtain optimum results. Because of the wide variety of devices to be measured there can be no universal solution to the fixture design problem. In fact, in the limit, each device measurement is unique and requires its own tailored measurement system. A thorough treatment of the design problem is beyond the scope of this presentation but some general comments may be made.

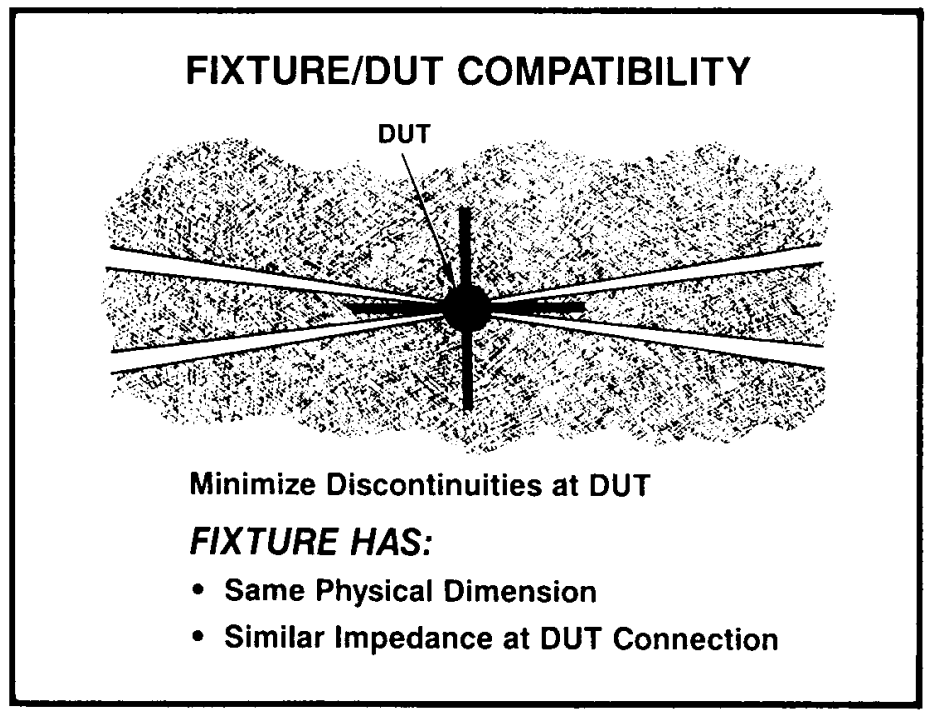

3983

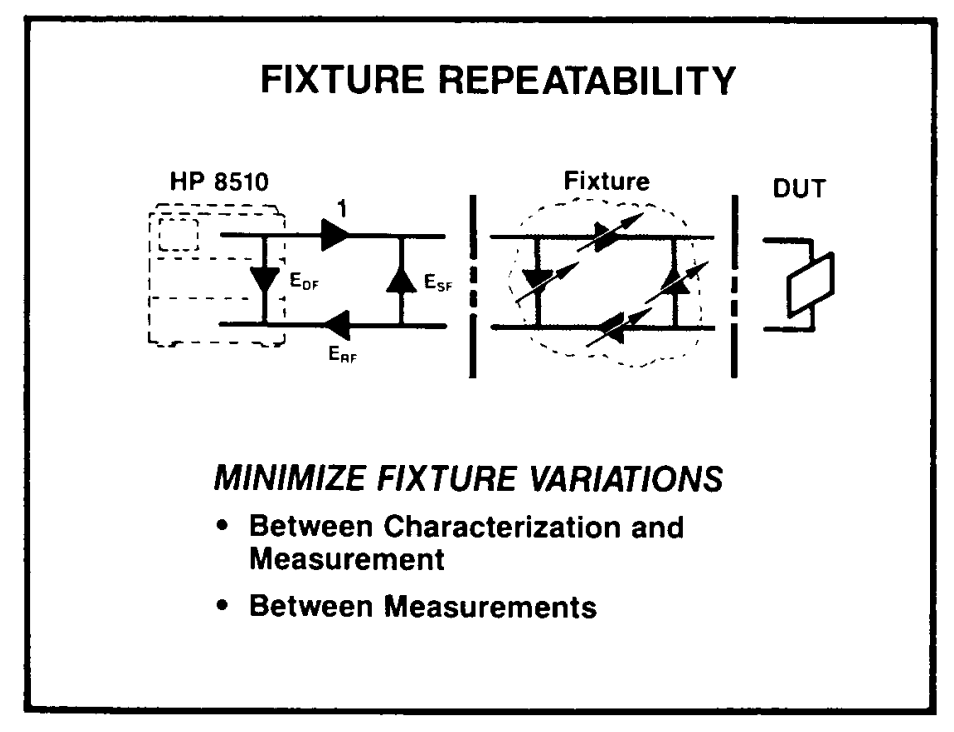

3984

\section{Compatibility With The DUT}

One goal of a fixture is to provide a Measurement environment for a DUT which is as much like the application environment as possible. This is especially desirable for devices which have performance which is strongly environment dependent. As an example, common lead impedance in transistors with low input or Output impedances can dramatically affect device performance in both a measurement fixture and an application. Also it is desirable to have a fixture which is optimized for the range of impedances being measured. In the case of very low impedance devices this may require a fixture which transforms the calibration impedance to the range of interest.

\section{Fixture Repeatability}

For an error corrected measurement (whether de-embedded or calibrated to the measurement plane) to be accurate, the fixtures characteristics must not change between the time the fixture is characterized and the time the measurement is made. Fixture repeatability establishes fundamental bounds for accuracy since nonrepeatable errors cannot be corrected. 


\section{Fixture Independence}

Because the error model for the HP 8510 requires it, the characteristics of the fixture must be independent of the device which is being measured. Although the fixture characterization using standards or "pseudostandards" may be accurate for measurements of a DUT which has the same value as a standard, it may be in error for all other values. This sort of error effectively distorts the Smith chart upon which the data is plotted.

This figure illustrates the dependence or coupling which can exist across the measurement plane between the discontinuity due to the DUT and an additional discontinuity within the fixture. Such fixture discontinuities need to be made small enough and be separated far enough from the measurement plane to allow a linear model to adequately describe the fixture.

\section{Ability To \\ Characterize The Fixture}

Since a DUT measurement requires that a fixture be characterized, enough standards or pseudostandards Must exist to allow this. This may be accomplished by conventional calibration with device plane standards or by measuring the standards or psdueostandards and using computer optimization to fit the data to a model.

\section{Convenience And Practicality}

The previous items may be necessary but are not sufficient to provide useful measured data. The fixture must also have utility. For this to be the case, operation Must be reasonably convenient. It must not be necessary to constantly recharacterize the fixture and the characterization process needs to be acceptable. Multiple measurements of many hard to connect standards and an involved calculation process may be unacceptable. Additionally the DUT itself Must be reasonably easy to install in the fixture. Arrangements which require special preparation of the DUT or a mounting technique which renders a device unavailable for future use may be unacceptable too.

This list of considerations is not intended to be exhaustive, but rather, a starting point for fixture design.

\section{ILLUSTRATION OF FIXTURE/ DUT DEPENDENCE}

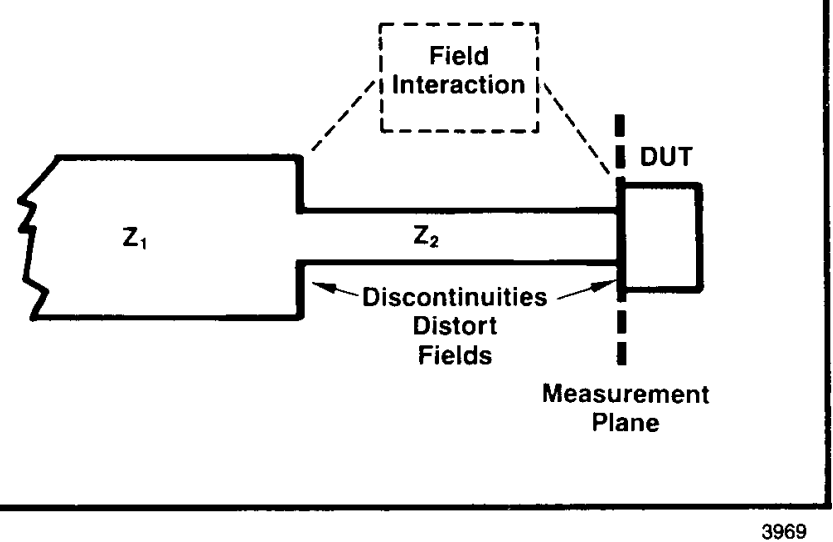

FIXTURE CHARACTERIZATION

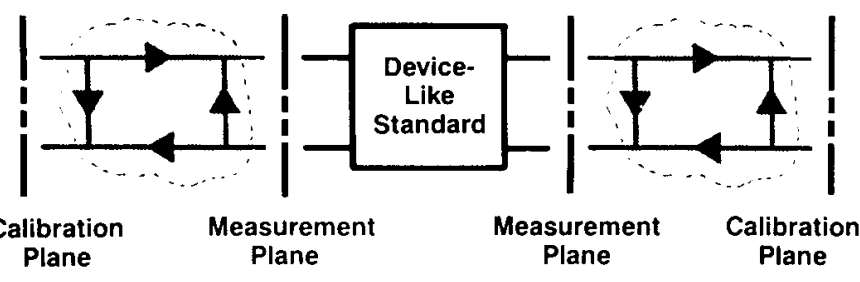

- Device-Like Standards or Pseudo-Standards Must Exist to Characterize the Fixture 


\section{EMBEDDING}

- The Process of De-Embedding is Useful to Reveal Actual DUT Characteristics

- The Possibility Exists of Mathematically Embedding the DUT in Hypothetical Networks to Reveal the Behavior of a Complete Circuit

3970

\section{DE-EMBEDDING TO GET ACTUAL DUT DATA}

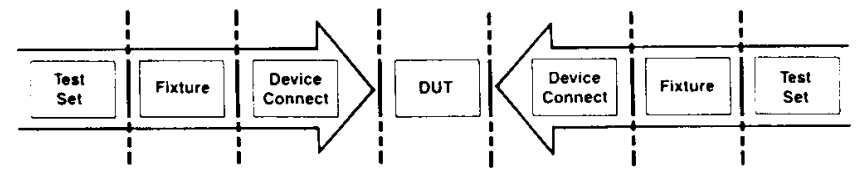

Planes of Partition

- Test Set

- Fixture

- Device Connections

\section{EMBEDDING}

So far in this discussion the emphasis has been on a means of modifying HP 8510 error terms to de-embed the DUT from a physically real fixture to provide a measurement plane right at the DUT, thus allowing the HP 8510 to operate exactly as though it had been calibrated there. This is desirable to enable measurement of the DUT itself, without fixturing effects. Once this has been achieved however, an additional possibility exists; that of using a similar process to include the effects of hypothetical two-port networks in the measurement of the DUT. This technique, called embedding, enables a device or circuit to be viewed as though it were actually embedded in Such networks. In essence this provides a blend of the domains of measurement and design. A real device can be operated with real world conditions, bias,

temperature, input power and the like, but be viewed as though it were in a circuit which does not really exist.

The de-embedding process can be viewed as a modification of the ANA vantage point, effectively moving it to different physically real planes in tile fixture. Such planes are here called planes of partition. They represent real planes which are orthogonal to the direction of energy flow in the fixture transmission medium. They may or may not be physically accessible.

A multitiered de-embedding process moves the measurement plane from the calibration plane toward the DUT. The measurement plane is moved from one plane of partition to the next ending at the device measurement plane. 
As a DUT is de-embedded from a realworld (causal) network, the measurement plane moves toward the DUT.

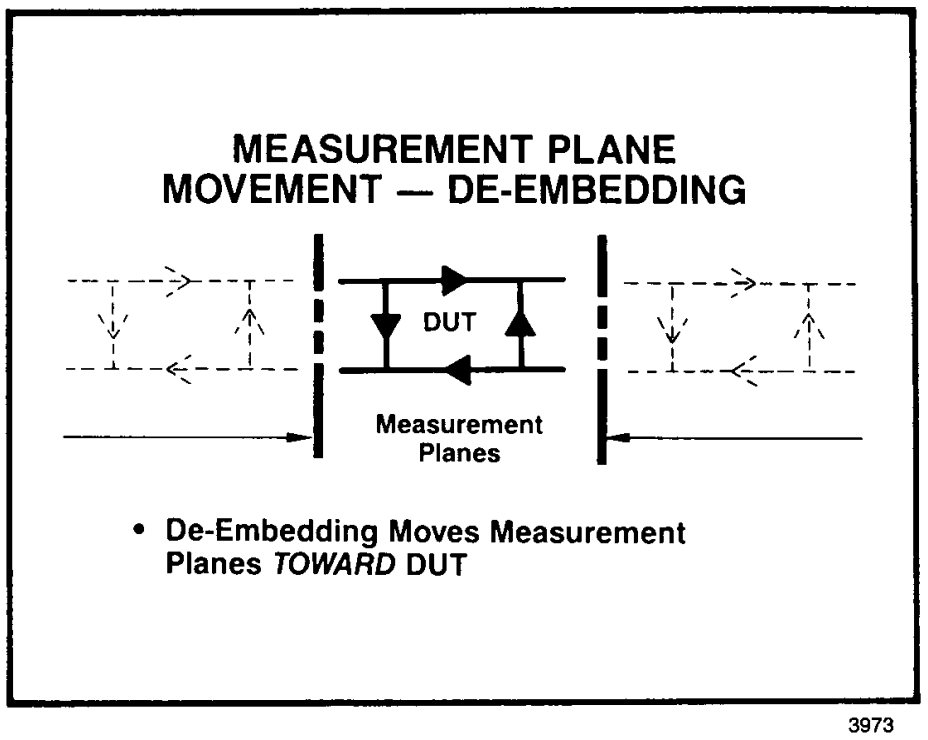

The embedding process similarly moves the measurement vantage point relative to the DUT. In this case, however, the vantage point does not need to have a physical reality and is therefore called a measurement frame instead of a measurement plane. In a sense it is a frame of reference frorn which to view, the DUT. It is not required to be physically realizable, and in fact, the planes of partition from the de-embedding process are a physically real subset of all measurement frames. A common example of measurement frame data is observed when a conventional ANA calibration is performed with an improperly connected standard thruline. Subsequent data taken of the same thruline used to calibrate, but properly connected, can reveal data implying physically impossible characteristics, a thruline with power gain, for example.

As a DUT is embedded in a realworld (causal) network, the measurement frame moves away from the DUT.

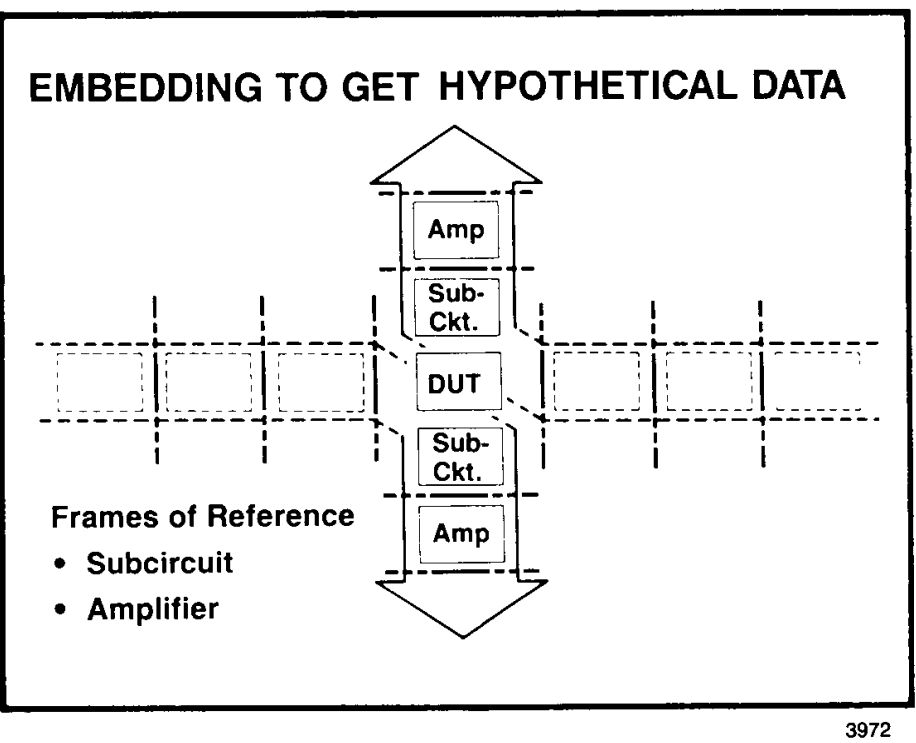

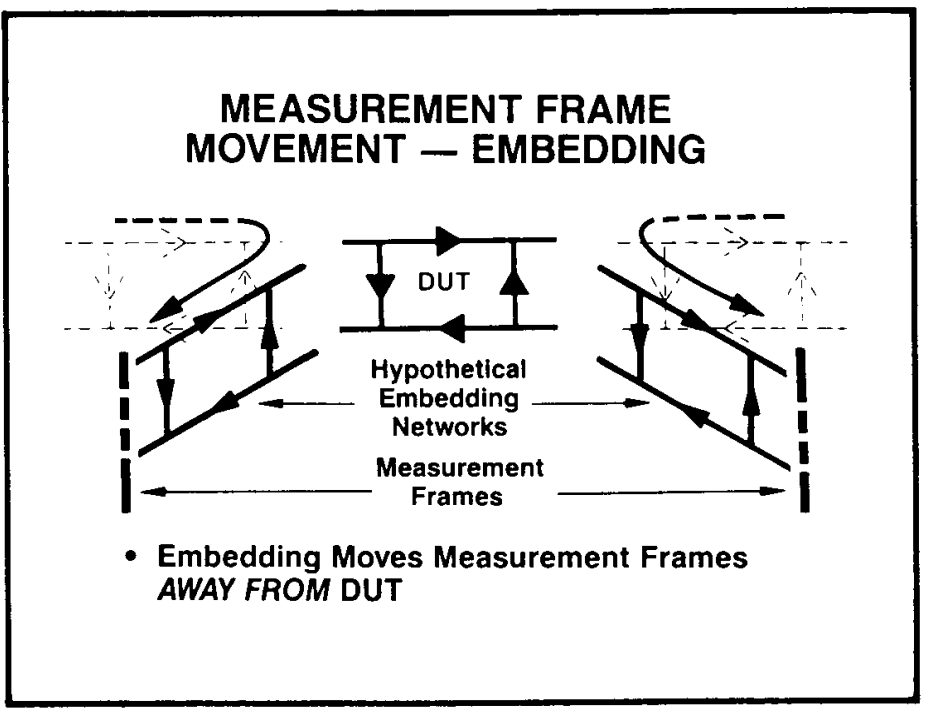




\section{EMBEDDING PROCESS}

- De-Embedding Algorithm May Be Used to EMBED a Fictitious Network.*

"Appendix B

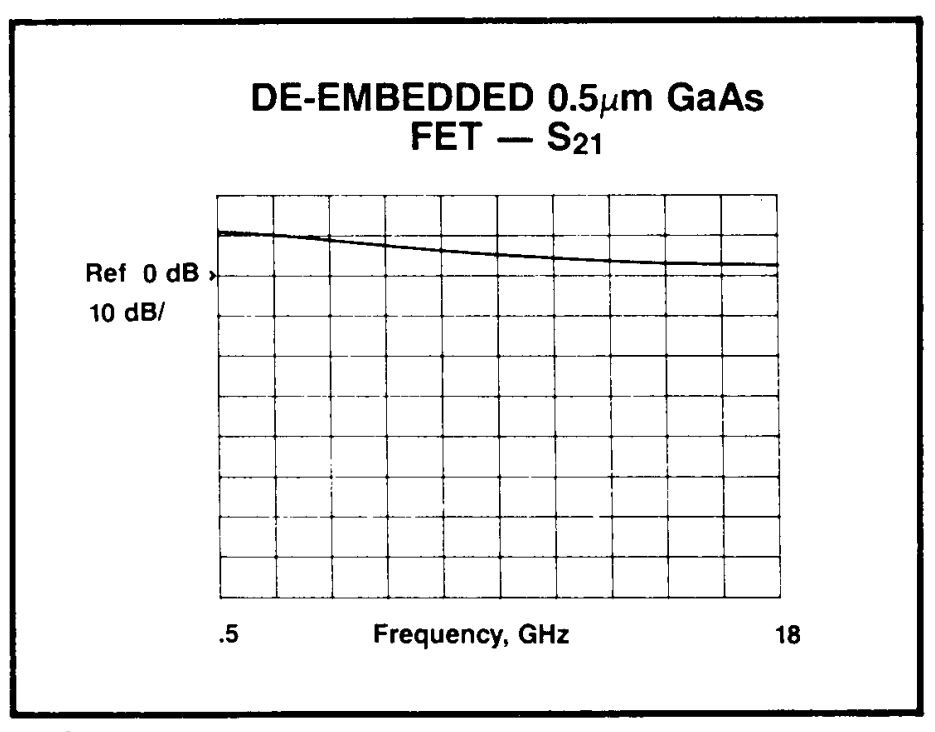

3976

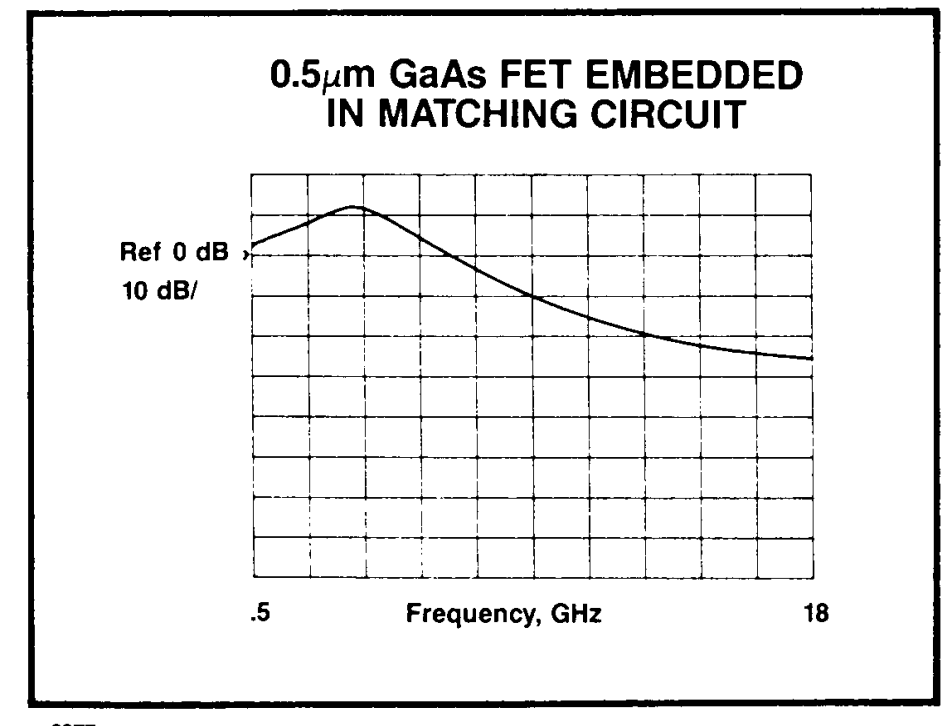

3977
In the preceding discussion of de-embedding and embedding the distinction was based on the direction of measurement plane or frame movement when Casual networks were being considered. If all networks are considered, including those which are noncausal, the distinction vanishes. In fact the process of de-embedding the DUT from a given network ca $\mathrm{n}$ be shown to give results identical to the process of' embedding the DUT in an "antinetwork", where the antinetwork has the characteristic that its cascade with the original network gives an identity network. If the original network was nonidentity and causal then the antinetwork will be noncausal. This allows embedding to be performed by Using the de-embedding algorithm but modifying the error terms Using the terms from the antinetwork. Equations for Calculating the antinetwork are in Appendix B.

To demonstrate the process, the following figures show the measurement of a transistor embedded in hypothetical matching and filter networks. This allows the transistor to he inserted into the fixture, measured as a function of bias and the results of a complete amplifier circuit with that particular device to be displayed. In this case, the effects of device type or temperature on the parameters of the complete but as yet unbuilt amplifier could also have been observed.

This figure shows the fully de-embedded insertion gain measurement of it 0.5 micron gate length gallium arsenide field effect transistor. The gain decreases monotonically with increasing frequency.

This figure shows the ANA error coefficients from the previous figure modified to embed the DUT in hypothetical matching networks. These networks can be seen to provide some additional gain at approximately $4 \mathrm{GHz}$. 
In this figure the previous ANA error coefficients have been modified to include the effects of hypothetical filter networks on the ANA ends of the previous matching Circuits. The only change among these three figures has been in the error coefficients which were provided for the ANA to correct the raw data. The DUT, fixture, ANA and the raw data taken by the analyzer internally at the data collection plane have remained unchanged. Only the measurement frame of the corrected data is different.

\section{$0.5 \mu \mathrm{m}$ GaAs FET EMBEDDED IN MATCHING/FILTER CIRCUIT}

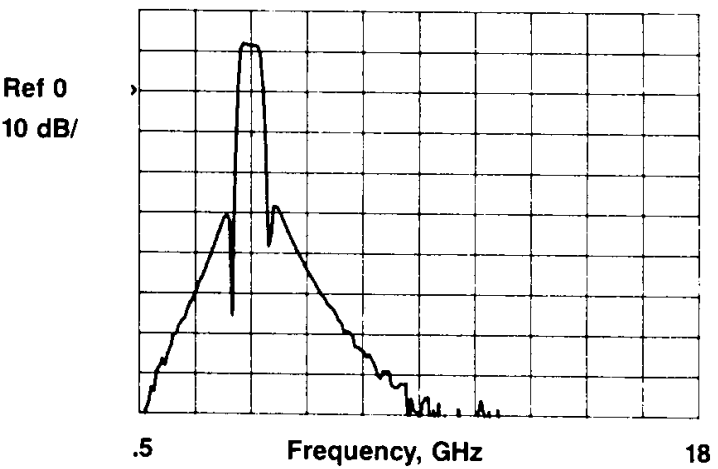

3978

This final figure demonstrates the blend of analysis and measurement which the technique allows. The properties of a physically real DUT can be examined as they relate to, and interact with, hypothetical networks. Here the bias point of the DUT is changed and the resulting "finished" amplifier performance can be seen relative to the DUT parameter changes in realtime.
"FINISHED" AMPLIFIER RESPONSE VS. BIAS

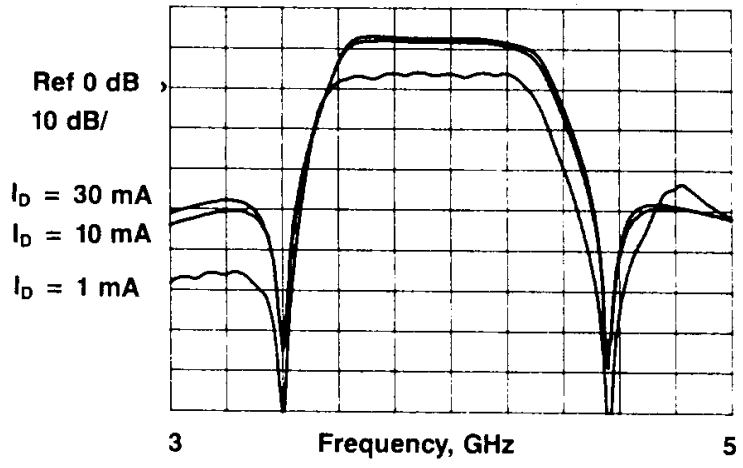




\section{APPENDIX A \\ HP 8510 A De-embedding Equations}

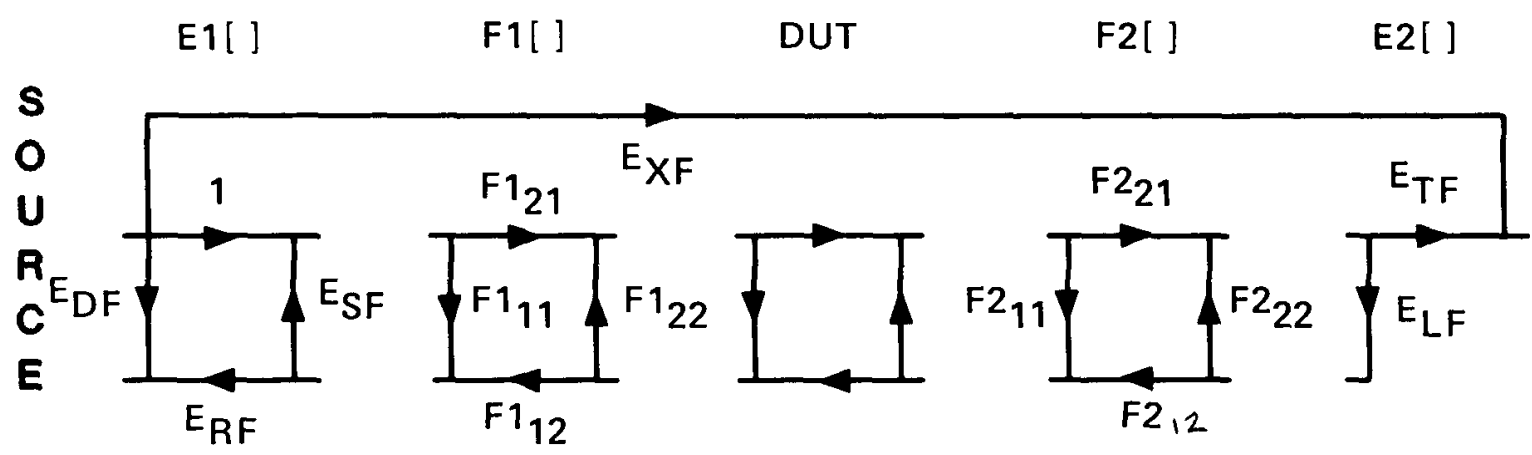

Figure A1. Two-Port Forward Direction Flow Diagram

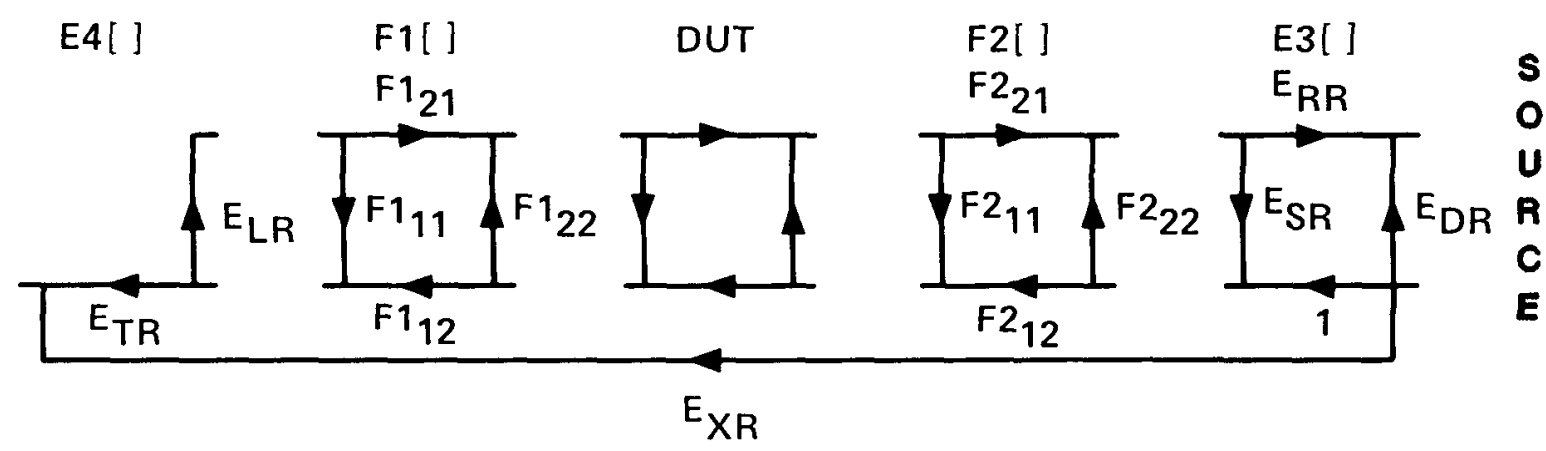

Figure A2. Two-Port Reverse Direction Flow Diagram

The figures show the 12 original two-port calibration $\mathrm{E}_{\text {terms }}{ }^{4}$ labeled $\mathrm{E}_{[}\left[\mathrm{]}-\mathrm{E}_{4}[\mathrm{]}\right.$, which are to be cascaded with the fixture error terms, $\mathrm{F}_{1}[]$ and $\mathrm{F}_{2}\left[\mathrm{]}\right.$, to provide modified terms, $\mathrm{E}^{\prime}$, for use by the HP 8510. It should be noted that the $F_{1}[]$ and $F_{2}[$ ] terms correspond to networks physically located on the port 1 and port 2 sides of the DUT respectively while the $\mathrm{E}$ terms do not have a direct physical representation, This is because the unity $\mathrm{E}_{\mathrm{l}}[]$ and $\mathrm{E}_{3}[\mathrm{]}$ forward transmission terms require normalization of the $\mathrm{E}_{\mathrm{rf}}, \mathrm{E}_{\mathrm{tf}}, \mathrm{E}_{\mathrm{rr}}$ and $\mathrm{E}_{\mathrm{tr}}$ terms. This normalization causes source side characteristics to be included in the opposite side's transmission tracking term.

Because the de-embedding equations for the forward and reverse directions are symmetrical, only the forward direction is developed here. The reverse direction equations are obtained by appropriate subscript changes of the forward direction equations. 
The E and F terms on the same side of the DUT are first cascaded using either topographical or analytical rnethods6. The result is an intermediate I matrix on each side of the DUT, $\mathrm{I}_{1}[]$ and $\mathrm{I}_{2}[\mathrm{]}$.

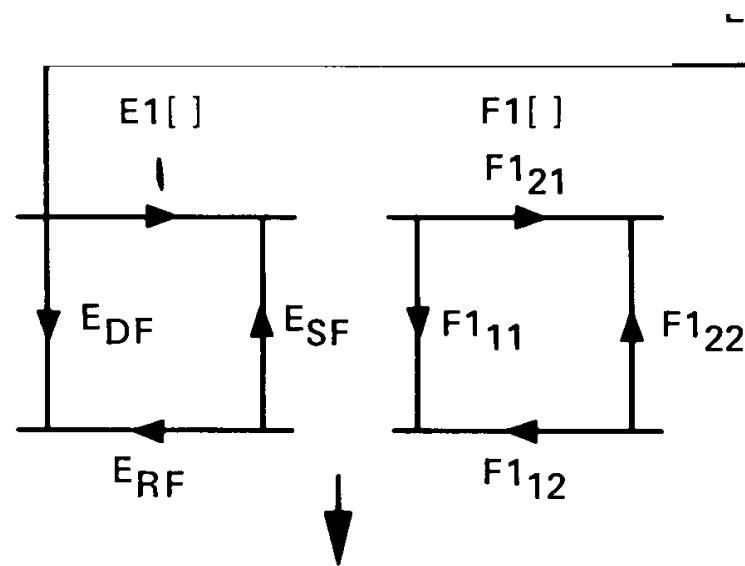

11[]

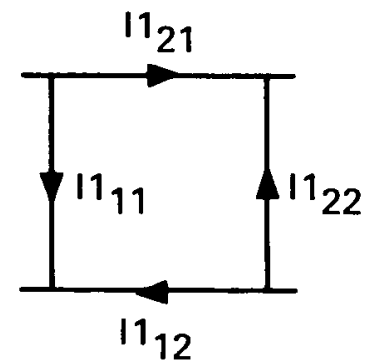

ᄂXF
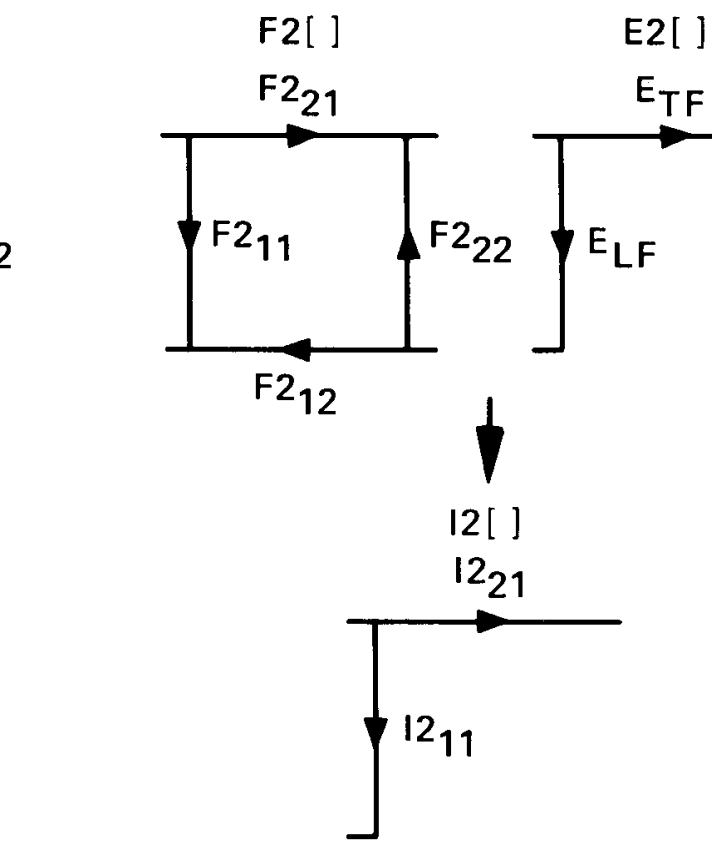

Figure A3. Forward Direction Cascading

$\mathrm{I}_{11}=\mathrm{E}_{\mathrm{df}}+\left(\mathrm{E}_{\mathrm{rf}} \mathrm{F} 1_{11}\right) /\left(1-\mathrm{E}_{\mathrm{sf}} \mathrm{Fl}_{11}\right)$

$\mathrm{I} 1_{12}=\left(\mathrm{E}_{\mathrm{rf}} \mathrm{F} 1_{12}\right) /\left(1-\mathrm{E}_{\mathrm{sf}} \mathrm{F} 1_{11}\right)$

$\mathrm{I} 1_{21}=\left(\mathrm{F} 1_{21}\right) /\left(1-\mathrm{E}_{\mathrm{sf}} \mathrm{F} 1_{11}\right)$

$\mathrm{I} 1_{22}=\mathrm{F} 1_{22}+\left(\mathrm{E}_{\mathrm{sf}} \mathrm{F} 1_{21} \mathrm{~F} 1_{12}\right) /\left(1-\mathrm{E}_{\mathrm{sf}} \mathrm{F} 1_{11}\right)$

$\mathrm{I} 2_{11}=\mathrm{F} 2_{11}+\left(\mathrm{E}_{\mathrm{lf}} \mathrm{F} 2_{21} \mathrm{~F} 2_{12}\right) /\left(1-\mathrm{E}_{\mathrm{lf}} \mathrm{F} 2_{22}\right)$

$\mathrm{I} 2_{21}=\left(\mathrm{E}_{\mathrm{tf}} \mathrm{F} 2_{21}\right) /\left(\mathrm{I}-\mathrm{E}_{\mathrm{lf}} \mathrm{F} 2_{22}\right)$

It is then necessary to adjust the I[ ] transmission terms to the form required by tile network analyzer internal error model (E terms). This is done by setting E1 ${ }_{21}$ to 1 and placing the product of $\mathrm{I} 1_{21}$ and $\mathrm{I} 1_{12}$ into $\mathrm{E}_{\mathrm{rf}}^{\prime}$. Similarly, the forward tracking term, $\mathrm{E}_{\mathrm{tf}}^{\prime}$ is obtained by multiplying $\mathrm{I} 2_{21}$ by this same term, $\mathrm{I}_{21}$. This provides the network analyzer with transmission error terms which are normalized to the source side forward transmission term, $\mathrm{El}_{21}$.

$\mathrm{E} 1^{\prime}{ }_{21}=1$

$\mathrm{E}_{\mathrm{rf}}^{\prime}=\mathrm{E} 1_{12}^{\prime}=\mathrm{I} 1_{12} \mathrm{I} 1_{21}$ 
$\mathrm{E}_{\mathrm{tf}}^{\prime}=\mathrm{E} 2^{\prime}{ }_{21}=\mathrm{I} 1_{21} \mathrm{I}_{21}$

The other $\mathrm{E}^{\prime}\left[\right.$ ] terms are the same as the I[ ] terms and the isolation term, $\mathrm{E}_{\mathrm{xf}}^{\prime}$, needs no modification since it is outside of the cascading process.

The resultant 12 two-port de-embedding equations follow:

$$
\begin{aligned}
& \mathrm{E}_{\mathrm{df}}^{\prime}=\mathrm{E}_{\mathrm{df}}+\left(\mathrm{E}_{\mathrm{rf}} \mathrm{F} 1_{11}\right) /\left(1-\mathrm{E}_{\mathrm{sf}} \mathrm{F} 1_{11}\right) \\
& \mathrm{E}_{\mathrm{rf}}^{\prime}=\left(\mathrm{E}_{\mathrm{rf}} \mathrm{F} 1_{12} \mathrm{~F} 1_{21}\right) /\left(1-\mathrm{E}_{\mathrm{sf}} \mathrm{F} 1_{11}\right)^{2} \\
& \mathrm{E}_{\mathrm{sf}}^{\prime}=\mathrm{F} 1_{22}+\left(\mathrm{E}_{\mathrm{sf}} \mathrm{F} 1_{12} \mathrm{~F} 1_{21}\right) /\left(1-\mathrm{E}_{\mathrm{sf}} \mathrm{F} 1_{11}\right) \\
& \mathrm{E}_{\mathrm{lf}}^{\prime}=\mathrm{F} 2_{11}+\left(\mathrm{E}_{\mathrm{lf}} \mathrm{F} 2_{12} \mathrm{~F} 2_{21}\right) /\left(1-\mathrm{E}_{\mathrm{lf}} \mathrm{F} 2_{22}\right) \\
& \mathrm{E}_{\mathrm{tf}}^{\prime}=\left(\mathrm{E}_{\mathrm{tf}} \mathrm{F} 1_{21} \mathrm{~F} 2_{21}\right) /\left(\left(1-\mathrm{E}_{\mathrm{lf}} \mathrm{F} 2_{22}\right)\left(1-\mathrm{E}_{\mathrm{sf}} \mathrm{F} 1_{11}\right)\right) \\
& \mathrm{E}_{\mathrm{xf}}^{\prime}=\mathrm{E}_{\mathrm{xf}} \\
& \mathrm{E}_{\mathrm{dr}}^{\prime}=\mathrm{E}_{\mathrm{dr}}+\left(\mathrm{E}_{\mathrm{rr}}-\mathrm{F} 2_{22}\right) /\left(1-\mathrm{E}_{\mathrm{sr}} \mathrm{F} 2_{22}\right) \\
& \mathrm{E}_{\mathrm{rr}}^{\prime}=\left(\mathrm{E}_{\mathrm{rr}} \mathrm{F} 2_{12} \mathrm{~F} 2_{21}\right) /\left(1-\mathrm{E}_{\mathrm{sr}} \mathrm{F} 2_{22}\right)^{2} \\
& \mathrm{E}_{\mathrm{lr}}^{\prime}=\mathrm{F} 2_{11}+\left(\mathrm{E}_{\mathrm{sr}} \mathrm{F} 2_{12} \mathrm{~F} 2_{21}\right) /\left(\mathrm{E}_{\mathrm{lr}} \mathrm{F} 1_{12} \mathrm{~F} 1_{21}\right) /\left(1-\mathrm{E}_{\mathrm{sr}} \mathrm{F} 2_{22}\right) \\
& \mathrm{E}_{\mathrm{tr}}^{\prime}=\left(\mathrm{E}_{\mathrm{tr}} \mathrm{F} 1_{11} \mathrm{~F} 2_{12}\right) /\left(\left(1-\mathrm{E}_{\mathrm{lr}} \mathrm{F} 1_{11}\right)\left(1-\mathrm{E}_{\mathrm{sr}} \mathrm{F} 2_{22}\right)\right) \\
& \mathrm{E}_{\mathrm{xr}}^{\prime}=\mathrm{E}_{\mathrm{xr}}
\end{aligned}
$$

For one-port de-embedding, only $E_{d f}^{\prime}, E_{r f}^{\prime}, E_{s f}^{\prime}$ or $E_{d r}^{\prime}, E_{r r}^{\prime}, E_{s r}^{\prime}$ are used for port 1 or port 2 measurernents, respectively. 


\section{APPENDIX B}

\section{Using the De-embedding Algorithm to Embed a Network}

The distinction between de-embedding and embedding is made based on tile direction of measurement frame movement relative to the DUT. If tile network through which the measurement plane moves is real, that is, causal, then movement of tile measurement frame toward the DUT is termed de-embedding while movement away from the DUT is called embedding. If no restriction is placed on the network to be considered then the distinction between de-embedding and embedding based on direction of measurement frame movement disappears. It then is possible to use either algorithm for both the de-embedding and the embedding processes.

To use the de-ernbedding equations to embed a network, some "preconditioning" of the network to be embedded must first take place. Consider the following cascaded networks:

Figure Bl. Cascade of network and antinetwork

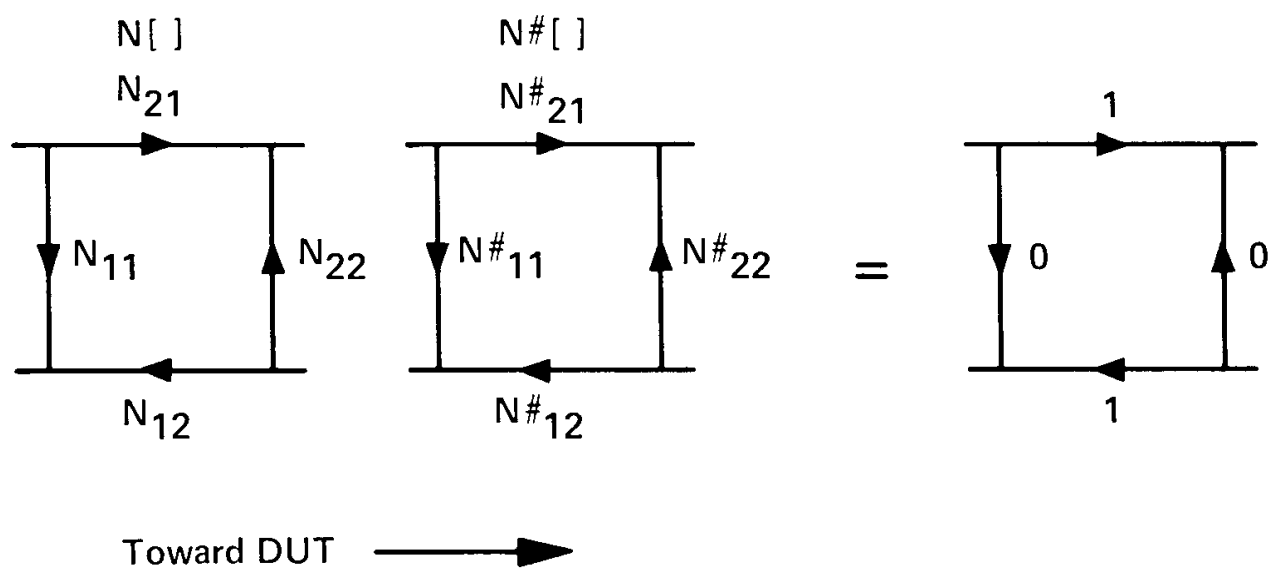

In Figure BI, N[ ] is intended to represent the network which is to be embedded while N:4[ ] is the corresponding "antinetwork". Since the cascade of these two networks is defined to be an identity network, it can be seen that one of the two will always be causal while the other is noncausal, except for the particular case where both are identity networks.

Now consider a two-tier de-embedding process of these cascaded networks. It is clear that the result must be an unchanged measurement frame. After the first tier of de-embedding, the measurement frame has moved through the $\mathrm{N}[$ ] network and effectively de-embedded the measurement from it. After the second tier of de-embedding, the measurement frame will again have moved, this time through the antinetwork, $\mathrm{N}^{\#}[]$. The measurement frame is now on the right side or the cascaded networks, but because the cascade is defined to be an identity network, this is the same as a measurement frame on the left side of the cascade. Movement of the measurement frame toward the DUT (de-embedding) through the antinetwork, $N^{\#}[]$, is therefore the same as movement of the measurement frame away from the DUT (embedding) through the network, N[ ].

In order to embed a network, $\mathrm{N}[\mathrm{]}$, it is only necessary to calculate and de-embed the antinetwork $\mathrm{N}^{\#}[]$. 
To calculate the antinetwork, first write the cascading equations for the two networks.

$$
\begin{aligned}
& \mathrm{S}_{11}=\mathrm{N}_{11}+\left(\mathrm{N}_{21} \mathrm{~N}_{12} \mathrm{~N}_{11}^{\#}\right) /\left(1-\mathrm{N}_{22} \mathrm{~N}_{11}^{\#}\right) \\
& \mathrm{S}_{12}=\left(\mathrm{N}_{12} \mathrm{~N}_{12}^{\#}\right) /\left(1-\mathrm{N}_{22} \mathrm{~N}_{11}^{\#}\right) \\
& \mathrm{S}_{21}=\left(\mathrm{N}_{21} \mathrm{~N}^{\#}{ }_{21}\right) /\left(1-\mathrm{N}_{22} \mathrm{~N}_{11}^{\#}\right) \\
& \mathrm{S}_{22}=\mathrm{N}_{22}^{\#}+\left(\mathrm{N}_{12}^{\#} \mathrm{~N}_{21}^{\#} \mathrm{~N}_{22}\right) /\left(1-\mathrm{N}_{22} \mathrm{~N}_{11}^{\#}\right)
\end{aligned}
$$

Setting the resulting matrix, $\mathrm{S}[\mathrm{]}$ equal to an identity matrix,

$\mathrm{S}_{11}=\mathrm{S}_{22}=0$ and $\mathrm{S}_{21}=\mathrm{S}_{12}=1$

gives;

$0=\mathrm{N}_{11}+\left(\mathrm{N}_{21} \mathrm{~N}_{12} \mathrm{~N}_{11}^{\#}\right) /\left(1-\mathrm{N}_{22} \mathrm{~N}_{11}^{\#}\right)$

$1=\left(\mathrm{N}_{12} \mathrm{~N}_{12}^{\#}\right) /\left(1-\mathrm{N}_{22} \mathrm{~N}_{11}^{\#}\right)$

$1=\left(\mathrm{N}_{21} \mathrm{~N}^{\#}{ }_{21}\right) /\left(1-\mathrm{N}_{22} \mathrm{~N}_{11}^{\#}\right)$

$0=\mathrm{N}_{22}^{\#}+\left(\mathrm{N}_{12}^{\#} \mathrm{~N}_{21}^{\#} \mathrm{~N}_{22}\right) /\left(1-\mathrm{N}_{22} \mathrm{~N}_{11}^{\#}\right)$

Solving for $\mathrm{N}^{\#}{ }_{11}$ gives:

$\mathrm{N}^{\#}{ }_{11}=\mathrm{N}_{11} /\left(\mathrm{N}_{11} \mathrm{~N}_{22}-\mathrm{N}_{21} \mathrm{~N}_{12}\right)$

which may be used to solve;

$\mathrm{N}_{12}^{\#}=\left(1-\mathrm{N}_{22} \mathrm{~N}_{11}^{\#}\right) / \mathrm{N}_{12}$

$\mathrm{N}^{\#}{ }_{21}=\left(1-\mathrm{N}_{22} \mathrm{~N}_{11}^{\#}\right) / \mathrm{N}_{21}$

which may be used to get

$$
\mathrm{N}^{\#}{ }_{22}=\left(\mathrm{N}_{12}^{\#} \mathrm{~N}_{21}^{\#} \mathrm{~N}_{22}\right) /\left(\mathrm{N}_{22} \mathrm{~N}_{11}^{\#}-1\right)
$$

To embed a network, $\mathrm{N}\left[\mathrm{]}\right.$, first calculate the antinetwork, $\mathrm{N}^{\#}[\mathrm{]}$ using the above equations and then provide the results to the de-embedding routine as the network to be de-embedded. De-embedding of this antinetwork, $\mathrm{N}^{\#}[]$ is the same as embedding the original network $\mathrm{N}[\mathrm{]}$. 


\section{REFERENCES}

[1] D. Rytting, "An Analysis of Vector Measurement Accuracy Enhancement Techniques", Hewlett-Packard, RF\&MW Symposium 1980

[2] R. F. Bauer and P. Penfield Jr., "De-embeddingand Unterminating" IEEE Transactions on Microwave Theory and Techniques, vol. MTT-22, PP. 282-288, March 1974

[3] J. Fitzpatrick and J. Williams, "Measuring Noninsertable Devices With an ANA" Microwave Systems News, PP. 96-104, June 1981

[4] J. Fitzpatrick, "Error Models for Systems Measurement", Microwave Journal, May 1978

[5] R. Lane, "De-embedding Device Scattering Parameters", Microwave Journal, August 1984, p. 149

[6] Stephen F. Adam, Microwave Theory and Applications, Prentice-Hall 1969, PP 99-105

[7] Bianco, Parodi, Ridella and Selvaggi, "Launcher and Microstrip Characterization", IEEE

Transactions on Instrumentation and Measurement, vol. IM-25, no. 4, December 1976 
